\title{
ISOMETRIES OF NONCOMMUTATIVE METRIC SPACES
}

\author{
EFTON PARK
}

(Communicated by Palle E. T. Jorgensen)

\begin{abstract}
A. Connes has shown that a unital $C^{*}$-algebra equipped with an unbounded Fredholm module can be viewed as a "noncommutative" metric space. In this paper, the author defines a notion of an isometry of a noncommutative metric space, and computes several examples.
\end{abstract}

For many years it has been fruitful to think of $C^{*}$-algebras as "noncommutative" topological spaces. However, until recently, there has not been a notion of a noncommutative metric space. In [1], Connes shows that for $C^{*}$-algebras, the appropriate notion of a metric is that of an unbounded Fredholm module.

Definition. Let $A$ be a unital $C^{*}$-algebra. An unbounded Fredholm module $(\mathscr{H}, D)$ over $A$ consists of

(i) a Hilbert space $\mathscr{H}$ and a representation of $A$ on $\mathscr{H}$;

(ii) an unbounded, self-adjoint operator $D$ on $\mathscr{H}$ such that:

(a) the set $\{a \in A:[D, a]$ is densely defined and extends to a bounded operator on $\mathscr{H}\}$ is norm dense in $A$.

(b) $\left(1+D^{2}\right)^{-1}$ is a compact operator.

To see why an unbounded Fredholm module should be thought of as a noncommutative analogue of a metric, consider the following prototypical example: let $M$ be a compact Riemannian manifold and let $A$ be the $C^{*}$-algebra of continuous, complex-valued functions on $M$. Let $S$ be a bundle of Clifford modules over $M$, and let $D$ be the associated operator of Dirac type on $L^{2}(M, S)$, the Hilbert space of $L^{2}$-sections of $S$. Then $A$ acts on $L^{2}(M, S)$ by multiplication and $\left(L^{2}(M, S), D\right)$ is an unbounded Fredholm module. Connes points out in [1] that the geodesic distance $d(p, q)$ between any two points $p$ and $q$ on $M$ can be computed by the following formula:

$$
d(p, q)=\sup \{|f(p)-f(q)|: f \in A,\|[D, f]\|=1\} .
$$

Thus we can recover the geodesic distance (and hence also the Riemannian metric) from the unbounded Fredholm module $\left(L^{2}(M, S), D\right)$.

Received by the editors April 5, 1993; presented in the Special Session on $C^{*}$-Algebras and Algebraic Topology, 873rd meeting of the AMS, Springfield, MO, March 21, 1992.

1991 Mathematics Subject Classification. Primary 46L85, 46L87, 46L40.

Key words and phrases. Noncommutative topology, noncommutative geometry, automorphisms of $C^{*}$-algebras. 
Research on noncommutative metric spaces is in its infancy. However, it is already clear from the work that has been done in [1] and [2] that this subject will be very important in the future. For this reason, the author has been interested in finding and studying the appropriate notion of isometry of a noncommutative metric space.

Definition. Let $A$ be a unital $C^{*}$-algebra and let $(\mathscr{H}, D)$ be an unbounded Fredholm module over $A$. The isometry group of $A$ with respect to $(\mathscr{H}, D)$ is denoted by $\operatorname{Iso}(A, \mathscr{H}, D)$, and is defined to be

$$
\begin{aligned}
& \text { Iso }(A, \mathscr{H}, D)=\{\phi \in \operatorname{Aut}(A): \text { there exists a unitary } U \text { on } \mathscr{H} \text { such that } \\
& \left.\qquad U D=D U \text { and } \phi(a)=U^{*} a U \text { for every } a \in A\right\} .
\end{aligned}
$$

We will call the elements of $\operatorname{Iso}(A, \mathscr{H}, D)$ isometries with respect to $(\mathscr{H}, D)$; the justification for this name comes from the following result, which we shall prove in the next section.

Theorem. Let $M$ be a compact oriented manifold, equipped with a Riemannian metric $g$, let $L^{2}\left(\Lambda^{*}(M)\right)$ be the Hilbert space of $L^{2}$-forms on $M$, and let $d+d^{*}$ be the deRham operator on $L^{2}\left(\Lambda^{*}(M)\right)$. Then $\operatorname{Iso}\left(C(M), L^{2}\left(\Lambda^{*}(M)\right), d+d^{*}\right)$ is naturally isomorphic to $\operatorname{Iso}(M, g)$.

In this paper, we will compute Iso $(A, \mathscr{H}, D)$ for several naturally occurring unbounded Fredholm modules $(\mathscr{H}, D)$. The author hopes that these examples will show that the isometry group of an unbounded Fredholm module is an interesting object worthy of further study, and that the following concrete computations will shed light upon what happens generally.

\section{The COMmutative CASE}

Let $M$ be a compact, oriented manifold, and let $g$ be a Riemannian metric on $M$. Let $L^{2}\left(\Lambda^{*}(M)\right)$ be the Hilbert space of complex $L^{2}$-forms on $M$, and let $d+d^{*}$ be the deRham operator on $L^{2}\left(\Lambda^{*}(M)\right)$. Then $C(M)$ acts on $L^{2}\left(\Lambda^{*}(M)\right)$ by pointwise multiplication, and $\left(L^{2}\left(\Lambda^{*}(M)\right), d+d^{*}\right)$ is an unbounded Fredholm module over $C(M)$. The following theorem shows that Iso $\left(C(M), L^{2}\left(\Lambda^{*}(M)\right), d+d^{*}\right)$ can be naturally identified with the group Iso $(M, g)$ of isometries of $M$ with respect to $g$. However, before we prove the theorem, let us state a lemma that will be useful in the sequel.

Lemma 1.1. Let $(\mathscr{H}, D)$ be an unbounded Fredholm module over a unital $C^{*}$ algebra $A$, and suppose $\phi$ is in $\operatorname{Iso}(A, \mathscr{H}, D)$. If $a \in A$ has the property that $[D, a]$ is bounded, then $[D, \phi(a)]$ is also bounded, and $\|[D, \phi(a)]\|=\|[D, a]\|$. Proof. Choose a unitary $U$ on $\mathscr{H}$ that commutes with $D$ and implements $\phi$. Then if $[D, a]$ is bounded, so is $U^{*}[D, a] U=\left[D, U^{*} a U\right]=[D, \phi(a)]$. Furthermore, $\|[D, a]\|=\left\|U^{*}[D, a] U\right\|=\left\|\left[D, U^{*} a U\right]\right\|=\|[D, \phi(a)]\|$.

Theorem 1.2. Iso $\left(C(M), L^{2}\left(\Lambda^{*}(M)\right), d+d^{*}\right) \cong \operatorname{Iso}(M, g)$.

Proof. Define $\eta: \operatorname{Homeo}(M) \longrightarrow \operatorname{Aut}(C(M))$ by $\eta(\Phi)(f)=f \circ \Phi^{-1}$. It is well known that this map is an isomorphism; we shall show that $\eta$ restricts to an isomorphism from Iso $(M, g)$ to $\operatorname{Iso}\left(C(M), L^{2}\left(\Lambda^{*}(M)\right), d+d^{*}\right)$.

For any isometry $\Psi$ of $(M, g)$, define $U_{\Psi}: L^{2}\left(\Lambda^{*}(M)\right) \longrightarrow L^{2}\left(\Lambda^{*}(M)\right)$ by $\left(U_{\Psi}\right) \omega=\left(\Psi^{-1}\right)^{*}(\omega)$. It is easy to check that $\left(U_{\Psi}\right)^{*}=U_{\Psi-1}$, so $U_{\Psi}\left(U_{\Psi}\right)^{*}=$ 
$\left(U_{\Psi}\right)^{*} U_{\Psi}=I$, and thus $U_{\Psi}$ is unitary. Given $f \in C(M)$, let $M_{f}: L^{2}\left(\Lambda^{*}(M)\right)$ $\longrightarrow L^{2}\left(\Lambda^{*}(M)\right)$ denote pointwise multiplication by $f$. Then

$$
U_{\Psi} M_{f} U_{\Psi}^{*}=M_{(f \circ \Psi-1)}
$$

A routine calculation shows that $U_{\Psi}$ commutes with exterior differentiation, and since $\Psi$ is an isometry, $U_{\Psi}$ commutes with $* d *$ as well, where $*$ is the Hodge star. Thus $U_{\Psi}$ commutes with $d+d^{*}$, and therefore $\eta(\Psi)$ is in Iso $\left(C(M), L^{2}\left(\Lambda^{*}(M)\right), d+d^{*}\right)$ for every isometry $\Psi$ of $(M, g)$.

To show that $\eta$ maps Iso $(M, g)$ onto $\operatorname{Iso}\left(C(M), L^{2}\left(\Lambda^{*}(M)\right), d+d^{*}\right)$, take $\phi$ in Iso $\left(C(M), L^{2}\left(\Lambda^{*}(M)\right), d+d^{*}\right)$, and choose $\Phi \in \operatorname{Homeo}(M)$ so that $\eta\left(\Phi^{-1}\right)=\phi$. Let $p$ and $q$ be any two points in $M$. We use Lemma 1.1, the geodesic distance formula mentioned in the introduction, and the fact that $\phi$ is an automorphism to get

$$
\begin{aligned}
d(\Phi(p), \Phi(q)) & =\sup \{|f(\Phi(p))-f(\Phi(q))|: f \in C(M),\|[D, f]\|=1\} \\
& =\sup \{|\phi(f)(p)-\phi(f)(q)|: f \in C(M),\|[D, f]\|=1\} \\
& =\sup \{|\phi(f)(p)-\phi(f)(q)|: \phi(f) \in C(M),\|[D, \phi(f)]\|=1\} \\
& =\sup \{|g(p)-g(q)|: g \in C(M),\|[D, g]\|=1\} \\
& =d(p, q) .
\end{aligned}
$$

Thus $\Phi$ is an isometry with respect to geodesic distance. This implies that $\Phi$ leaves $g$ fixed [3, p. 61], so $\Phi$ is an isometry. Hence $\Phi^{-1}$ is also an isometry, and therefore $\eta: \operatorname{Iso}(M, g) \rightarrow \operatorname{Iso}\left(C(M), L^{2}\left(\Lambda^{*}(M)\right), d+d^{*}\right)$ is an isomorphism.

Remark. The group Iso $(M, g)$ is usually endowed with the compact-open topology. If we use the isomorphism from Theorem 1.2 to push forward the topology on $\operatorname{Iso}(M, g)$ to $\operatorname{Iso}\left(C(M), L^{2}\left(\Lambda^{*}(M)\right), d+d^{*}\right)$, we get the topology of pointwise convergence.

\section{REDUCED GROUP $C^{*}$-ALGEBRAS}

Let $\Gamma$ be a discrete group, and let $C_{r}^{*}(\Gamma)$ denote the reduced group $C^{*}$ algebra of $\Gamma$. An important class of unbounded Fredholm modules over $C_{r}^{*}(\Gamma)$ arises in the following manner. Let $S$ be a set of generators of $\Gamma$, and let $L$ be the word length function with respect to $S$. We define an operator $D$ on $l^{2}(\Gamma)$ by requiring that $D\left(\delta_{x}\right)=L(x) \delta_{x}$ for each $x$ in $\Gamma$; here $\delta_{x}$ denotes the unit point mass at $x$. As long as there are words in $\Gamma$ of arbitrarily long length with respect to $S,\left(l^{2}(\Gamma), D\right)$ is an unbounded Fredholm module over $C_{r}^{*}(\Gamma)$ [1]. In this section, we record several results involving the isometry groups of these unbounded Fredholm modules.

Lemma 2.1. Suppose that $\phi$ is in $\operatorname{Iso}\left(C_{r}^{*}(\Gamma), l^{2}(\Gamma), D\right)$, and let $\tau$ denote the standard trace on $C_{r}^{*}(\Gamma)$. Then $\tau \circ \phi=\tau$.

Proof. Let $e$ denote the identity of $\Gamma$. Since $e$ is the only element of $\Gamma$ that has length zero, the kernel of $D$ is the one-dimensional subspace spanned by $\delta_{e}$. Let $U$ be a unitary that implements $\phi$ and commutes with $D$. Then $U$ also commutes with all the spectral projections of $D$, whence $U\left(\delta_{e}\right)=\lambda \delta_{e}$ for some complex number $\lambda$ of modulus 1 . Thus, for every $X$ in $C_{r}^{*}(\Gamma),(\tau \circ \phi)(X)=$ $\left\langle\phi(X)\left(\delta_{e}\right), \delta_{e}\right\rangle=\left\langle U^{*} X U\left(\delta_{e}\right), \delta_{e}\right\rangle=\left\langle X U\left(\delta_{e}\right), U\left(\delta_{e}\right)\right\rangle=\left\langle X\left(\lambda \delta_{e}\right), \lambda \delta_{e}\right\rangle=$ $\left\langle X\left(\delta_{e}\right), \delta_{e}\right\rangle=\tau(X)$. 
The next result gives a way to obtain elements of $\operatorname{Iso}\left(C_{r}^{*}(\Gamma), l^{2}(\Gamma), D\right)$.

Proposition 2.2. Let $\Gamma$ be a finitely generated discrete group, and let $S$ be a finite set of generators of $\Gamma$. Suppose that $\alpha$ is an automorphism of $\Gamma$ that maps $S^{ \pm}=S \cup S^{-1}$ to $S^{ \pm}$, and suppose that $\chi$ is a homomorphism from $\Gamma$ to the circle group $\mathbb{T}$. Then the map $\lambda_{z} \mapsto \chi(z) \lambda_{\alpha(z)}$ extends to an element of Iso $\left(C_{r}^{*}(\Gamma), l^{2}(\Gamma), D\right)$.

Proof. Define an operator $U$ on $l^{2}(\Gamma)$ by the formula

$$
U\left(\delta_{x}\right)=\overline{\chi\left(\alpha^{-1}(x)\right)} \delta_{\alpha^{-1}(x)} .
$$

It is easy to check that $U^{*}\left(\delta_{x}\right)=\chi(x) \delta_{\alpha(x)}$ and $U U^{*}=U^{*} U=I$, so $U$ is unitary. Define $\phi: l^{2}(\Gamma) \longrightarrow l^{2}(\Gamma)$ by $\phi(T)=U^{*} T U$. Then $\phi\left(\lambda_{x}\right)=\chi(x) \lambda_{\alpha(x)}$ for every $x$ in $\Gamma$, and therefore $\phi$ maps $C_{r}^{*}(\Gamma)$ to $C_{r}^{*}(\Gamma)$. A similar computation shows that $\phi^{-1}$ also sends $C_{r}^{*}(\Gamma)$ to itself, so $\phi$ is an automorphism of $C_{r}^{*}(\Gamma)$.

To show that $\phi$ is an isometry of $\left(l^{2}(\Gamma), D\right)$, we verify that $U$ commutes with $D$, or equivalently (since $D$ is self-adjoint), that $U^{*}$ commutes with $D$. Now, since $U^{*} D\left(\delta_{x}\right)=L(x) U^{*}\left(\delta_{x}\right)=L(x) \chi(x)\left(\delta_{\alpha(x)}\right)$ and $D U^{*}\left(\delta_{x}\right)=$ $\chi(x) D\left(\delta_{\alpha(x)}\right)=L(\alpha(x)) \chi(x)\left(\delta_{\alpha(x)}\right)$, it suffices to show that $L(\alpha(x))=L(x)$ for all $x \in \Gamma$. Suppose $L(x)=n$, and choose $n$ elements $g_{1}, g_{2}, \ldots, g_{n}$ in $S^{ \pm}$such that $x=g_{1} g_{2} \ldots g_{n}$. Then $\alpha(x)=\alpha\left(g_{1}\right) \alpha\left(g_{2}\right) \ldots \alpha\left(g_{n}\right)$, and hence $L(\alpha(x)) \leq L(x)$. On the other hand, since $S^{ \pm}$is a finite set, $\alpha$ maps $S^{ \pm}$onto itself, and so if $\alpha(x)=h_{1} h_{2} \ldots h_{m}$ for some $h_{1}, h_{2}, \ldots, h_{m}$ in $S^{ \pm}$, then $x=$ $\alpha^{-1}\left(h_{1}\right) \alpha^{-1}\left(h_{2}\right) \ldots \alpha^{-1}\left(h_{m}\right)$, whence $L(x) \leq L(\alpha(x))$. Hence $L(\alpha(x))=L(x)$, and therefore $\phi$ is in Iso $\left(C_{r}^{*}(\Gamma), l^{2}(\Gamma), D\right)$.

Proposition 2.2 shows that $\operatorname{Iso}\left(C_{r}^{*}(\Gamma), l^{2}(\Gamma), D\right)$ is typically nontrivial. For certain groups $\Gamma$, all of the elements of $\operatorname{Iso}\left(C_{r}^{*}(\Gamma), l^{2}(\Gamma), D\right)$ are produced by the construction in Proposition 2.2:

Theorem 2.3. Let $\Gamma$ be a finitely generated discrete group, and let $S$ be a finite set of generators with the property that if $g_{1}, g_{2}, h_{1}, h_{2}$ are in $S^{ \pm}$and $g_{1} h_{1}=g_{2} h_{2} \neq e$, then $g_{1}=g_{2}$ and $h_{1}=h_{2}$. Let Aut $S^{ \pm}(\Gamma)$ be the group of automorphisms of $\Gamma$ that map $S^{ \pm}$to $S^{ \pm}$. Then Iso $\left(C_{r}^{*}(\Gamma), l^{2}(\Gamma), D\right)$ is isomorphic to a semidirect product $\operatorname{Hom}(\Gamma, \mathbb{T}) \rtimes \operatorname{Aut}_{S^{ \pm}}(\Gamma)$, with group operation defined by $\left(\chi_{1}, \alpha_{1}\right)\left(\chi_{2}, \alpha_{2}\right)=\left(\left(\chi_{1} \circ \alpha_{2}\right) \chi_{2}, \alpha_{1} \alpha_{2}\right)$.

Proof. In the proof of Proposition 2.2, we described a way to associate to each $\chi$ in $\operatorname{Hom}(\Gamma, \mathbb{T})$ and $\alpha$ in $\operatorname{Aut}_{S^{ \pm}}(\Gamma)$ an element of $\operatorname{Iso}\left(C_{r}^{*}(\Gamma), l^{2}(\Gamma), D\right)$; let $\beta: \operatorname{Hom}(\Gamma, \mathbb{T}) \rtimes \operatorname{Aut}_{S^{ \pm}}(\Gamma) \longrightarrow \operatorname{Iso}\left(C_{r}^{*}(\Gamma), l^{2}(\Gamma), D\right)$ denote this map. Direct computation shows that $\beta$ is a group homomorphism. Clearly $\beta$ is injective, so all that remains is to show that $\beta$ is surjective.

Choose $\phi$ in $\operatorname{Iso}\left(C_{r}^{*}(\Gamma), l^{2}(\Gamma), D\right)$, and $g \neq e$ in $S^{ \pm}$. Expand $\phi\left(\lambda_{g}\right)\left(\delta_{e}\right)$ in terms of the standard orthonormal basis for $l^{2}(\Gamma): \phi\left(\lambda_{g}\right)\left(\delta_{e}\right)=\sum_{x \in \Gamma} c_{x} \delta_{x}$, where $c_{x}=\left\langle\phi\left(\lambda_{g}\right)\left(\delta_{e}\right), \delta_{x}\right\rangle$. Since $\lambda_{g}$ is unitary, so is $\phi\left(\lambda_{g}\right)$, and therefore

$$
1=\left\|\delta_{e}\right\|_{2}^{2}=\left\|\phi\left(\lambda_{g}\right)\left(\delta_{e}\right)\right\|_{2}^{2}=\sum_{x \in \Gamma}\left|c_{x}\right|_{2}^{2}
$$

Using Lemma 1.1, we also get

$$
1=\left\|\left[D, \lambda_{g}\right]\right\|^{2}=\left\|\left[D, \phi\left(\lambda_{g}\right)\right]\right\|^{2} \geq\left\|\left[D, \phi\left(\lambda_{g}\right)\right]\left(\delta_{e}\right)\right\|_{2}^{2}=\sum_{x \in \Gamma} L(x)^{2}\left|c_{x}\right|^{2} .
$$


We have two relations involving the coefficients $c_{x}$ :

$$
\begin{gathered}
\sum_{x \in \Gamma}\left|c_{x}\right|^{2}=1, \\
\sum_{x \in \Gamma} L(x)^{2}\left|c_{x}\right|^{2} \leq 1 .
\end{gathered}
$$

Let $\tau$ denote the standard trace on $C_{r}^{*}(\Gamma)$. Then apply Lemma 2.1 to obtain $0=\tau\left(\lambda_{g}\right)=\tau\left(\phi\left(\lambda_{g}\right)\right)=\left\langle\phi\left(\lambda_{g}\right)\left(\delta_{e}\right), \delta_{e}\right\rangle=c_{e}$. Therefore the two relations above imply that $c_{x}=0$ unless $L(x)=1$; that is, $c_{x}$ is nonzero only if $x$ is in $S^{ \pm}$. Thus $\phi\left(\lambda_{g}\right)\left(\delta_{e}\right)=\sum_{x \in S^{ \pm}} c_{x} \delta_{x}$. Moreover, since each element of $C_{r}^{*}(\Gamma)$ is determined by its value on $\delta_{e}, \phi\left(\lambda_{g}\right)=\sum_{x \in S^{ \pm}} c_{x} \lambda_{x}$. Next,

$$
I=\phi\left(\lambda_{g}\right)\left(\phi\left(\lambda_{g}\right)\right)^{*}=\left(\sum_{x \in S^{ \pm}}\left|c_{x}\right|^{2}\right) I+\sum_{\substack{x, y \in S^{ \pm} \\ x \neq y}} c_{x} \bar{c}_{y} \lambda_{x y^{-1}},
$$

and therefore,

$$
\sum_{\substack{x, y \in S^{ \pm} \\ x \neq y}} c_{x} \bar{c}_{y} \lambda_{x y-1}=0
$$

By hypothesis, the group elements $x y^{-1}$ in the above sum are all distinct. This, combined with the requirement that $\sum_{x \in S^{ \pm}}\left|c_{x}\right|^{2}=1$, implies that $c_{x}$ is nonzero for exactly one element $x \in S^{ \pm}$, and $\left|c_{x}\right|=1$ for that $x$. Thus $\phi\left(\lambda_{g}\right)=c_{x} \lambda_{x}$ for some $\left|c_{x}\right|=1$ and $x \in S^{ \pm}$. Moreover, since $S$ generates $\Gamma$, we see that for any $y$ in $\Gamma$, there exists a complex number $c$ of norm 1 and an element $z$ in $\Gamma$ so that $\phi\left(\lambda_{y}\right)=c \lambda_{z}$.

Define maps $\alpha: \Gamma \longrightarrow \Gamma$ and $\chi: \Gamma \longrightarrow \mathbb{T}$ so that $\phi\left(\lambda_{x}\right)=\chi(x) \lambda_{\alpha(x)}$ for all $x \in \Gamma$. Then for all $x, y \in \Gamma, \chi(x y) \lambda_{\alpha(x y)}=\phi\left(\lambda_{x y}\right)=\phi\left(\lambda_{x}\right) \phi\left(\lambda_{y}\right)=$ $\chi(x) \chi(y) \lambda_{\alpha(x) \alpha(y)}$, whence $\chi$ and $\alpha$ are homomorphisms. The fact that $\phi$ is an automorphism of $C_{r}^{*}(\Gamma)$ implies that $\alpha$ is an automorphism of $\Gamma$. Finally, the computations we did earlier in the proof show that $\alpha$ maps $S^{ \pm}$to $S^{ \pm}$, so $\alpha \in \operatorname{Aut}_{S^{ \pm}}(\Gamma)$. Clearly $\beta(\chi, \alpha)=\phi$, so $\beta$ is surjective and defines an isomorphism between $\operatorname{Hom}(\Gamma, \mathbb{T}) \rtimes \operatorname{Aut}_{S^{ \pm}}(\Gamma)$ and $\operatorname{Iso}\left(C_{r}^{*}(\Gamma), l^{2}(\Gamma), D\right)$.

There is a variety of groups that admit generating sets $S$ satisfying the hypotheses of Theorem 2.3.

Example 2.4. $F_{n}$, the free group on $n$ generators $g_{1}, g_{2}, \ldots, g_{n} ; S=$ $\left\{g_{1}, g_{2}, \ldots, g_{n}\right\}$.

Every automorphism $\alpha$ of $F_{n}$ is determined by its values on the generators. Since we require that $\alpha$ map $S^{ \pm}$to $S^{ \pm}$, there exists a permutation $\sigma$ in $S_{n}$, the symmetric group on $n$ letters, with the property that $\alpha\left(g_{i}\right)=g_{\sigma(i)}^{k_{i}}$, where $k_{i}= \pm 1$. Therefore Aut $_{S^{ \pm}}\left(F_{n}\right) \cong\left(\mathbb{Z}_{2}\right)^{n} \rtimes S_{n}$.

Since $F_{n}$ is free, $\operatorname{Hom}\left(F_{n}, \mathbb{T}\right) \cong \mathbb{T}^{n}$, and thus

$$
\operatorname{Iso}\left(C_{r}^{*}\left(F_{n}\right), l^{2}\left(F_{n}\right), D\right) \cong \mathbb{T}^{n} \rtimes\left(\left(\mathbb{Z}_{2}\right)^{n} \rtimes S_{n}\right) .
$$

Example 2.5. The free product $\mathbb{Z}_{m} * \mathbb{Z}_{n}$ of finite cyclic groups, with $m, n>2$ and $m \neq n ; S=\left\{1_{m}, 1_{n}\right\}$. 
Since $m \neq n$, any automorphism of $\mathbb{Z}_{m} * \mathbb{Z}_{n}$ that takes $S^{ \pm}$to itself must map $1_{m}$ to $\pm 1_{m}$ and $1_{n}$ to $\pm 1_{n}$. Thus Aut $_{S^{ \pm}}\left(\mathbb{Z}_{m} * \mathbb{Z}_{n}\right) \cong \mathbb{Z}_{2} \oplus \mathbb{Z}_{2}$.

To determine $\operatorname{Hom}\left(\mathbb{Z}_{m} * \mathbb{Z}_{n}, \mathbb{T}\right)$, observe that $1_{m}$ and $1_{n}$ can only be sent to $m$ th and $n$th roots of unity, respectively. Therefore $\operatorname{Hom}\left(\mathbb{Z}_{m} * \mathbb{Z}_{n}, \mathbb{T}\right) \cong$ $\mathbb{Z}_{m} \oplus \mathbb{Z}_{n}$, and

$$
\text { Iso }\left(C_{r}^{*}\left(\mathbb{Z}_{m} * \mathbb{Z}_{n}\right), l^{2}\left(\mathbb{Z}_{m} * \mathbb{Z}_{n}\right), D\right) \cong\left(\mathbb{Z}_{m} \oplus \mathbb{Z}_{n}\right) \rtimes\left(\mathbb{Z}_{2} \oplus \mathbb{Z}_{2}\right) .
$$

Example 2.6. The free product $\mathbb{Z}_{m} * \mathbb{Z}_{m}$ of a finite cyclic group with itself, $m>2 ; S=\{1, \tilde{1}\}$. (We use the tilde to distinguish between elements of the two copies of $\mathbb{Z}_{m}$.)

There are eight automorphisms of $\mathbb{Z}_{m} * \mathbb{Z}_{m}$ that take $S^{ \pm}=\{1, \tilde{1},-1,-\tilde{1}\}$ to itself. Let $\alpha$ be the automorphism for which it is true that $\alpha(1)=-\tilde{1}$ and $\alpha(\tilde{1})=1$, and let $\beta$ be the automorphism given by $\beta(1)=\tilde{1}$ and $\beta(\tilde{1})=1$. Then $\alpha$ has order $4, \beta$ has order 2 , and $\beta \alpha=\alpha^{-1} \beta$. Therefore Aut $S_{S^{ \pm}}\left(\mathbb{Z}_{m} * \mathbb{Z}_{m}\right)$ is isomorphic to the dihedral group $D_{4}$.

By an argument similar to that used in Example 2.5, $\operatorname{Hom}\left(\mathbb{Z}_{m} * \mathbb{Z}_{m}, \mathbb{T}\right) \cong$ $\mathbb{Z}_{m} \oplus \mathbb{Z}_{m}$, so

$$
\operatorname{Iso}\left(C_{r}^{*}\left(\mathbb{Z}_{m} * \mathbb{Z}_{m}\right), l^{2}\left(\mathbb{Z}_{m} * \mathbb{Z}_{m}\right), D\right) \cong\left(\mathbb{Z}_{m} \oplus \mathbb{Z}_{m}\right) \rtimes D_{4} .
$$

\section{THE ROTATION ALGEBRAS}

Choose $\theta \in(0,1)$. The rotation $C^{*}$-algebra is the universal $C^{*}$-algebra $A_{\theta}$ generated by unitaries $V$ and $W$ satisfying the relation $W V=\lambda V W$, where $\lambda=\exp (2 \pi l \theta)$. In [1], Connes constructs an unbounded Fredholm module over $A_{\theta}$ which is analogous to the unbounded Fredholm module over $A_{0} \cong C\left(\mathbb{T}^{2}\right)$ defined by the Dirac operator on $\mathbb{T}^{2}$. We now describe this Fredholm module.

Define an inner product on $A_{\theta}$ by requiring that $\left\langle V^{k} W^{l}, V^{m} W^{n}\right\rangle=1$ if $k=m$ and $l=n$, and 0 otherwise. Let $L^{2}\left(A_{\theta}\right)$ denote the Hilbert space completion of $A_{\theta}$ with respect to this inner product, and define an unbounded operator $\partial$ on $L^{2}\left(A_{\theta}\right)$ by $\partial\left(V^{k} W^{l}\right)=2 \pi l(k+l l) V^{k} W^{l}$. Next, let $\mathscr{H}=$ $L^{2}\left(A_{\theta}\right) \oplus L^{2}\left(A_{\theta}\right)$. Then

$$
D=\left(\begin{array}{cc}
0 & \partial \\
\partial^{*} & 0
\end{array}\right)
$$

is an unbounded operator on $\mathscr{H}$. With the action $X \cdot\left(\begin{array}{l}Y \\ Z\end{array}\right)=\left(\begin{array}{l}X Y \\ X Z\end{array}\right)$ of $A_{\theta}$ on $\mathscr{H},(\mathscr{H}, D)$ is an unbounded Fredholm module over $A_{\theta}$.

In order to compute Iso $\left(A_{\theta}, \mathscr{H}, D\right)$, we first examine $D$ more closely. The eigenvalues of $D$ are numbers of the form $\pm 2 \pi \sqrt{m^{2}+n^{2}}$, and the eigenspace of $\pm 2 \pi \sqrt{m^{2}+n^{2}}$ is spanned by the set of vectors

$$
\left\{\left(\begin{array}{c}
\mp \sqrt{k^{2}+l^{2}} V^{k} W^{l} \\
(l k+l) V^{k} W^{l}
\end{array}\right): k^{2}+l^{2}=m^{2}+n^{2}\right\} .
$$

Suppose $\phi$ is in $\operatorname{Iso}\left(A_{\theta}, \mathscr{H}, D\right)$, and choose a unitary $U$ such that $U D=D U$ and such that $\phi(X)=U^{*} X U$ for all $X \in A_{\theta}$. For any element $X$ in $A_{\theta}$, $U^{*} X U\left(\begin{array}{l}I \\ 0\end{array}\right)=\left(\begin{array}{l}Y \\ 0\end{array}\right)$ for some $Y \in A_{\theta}$, and $U^{*} X U$ is completely determined by its value at $\left(\begin{array}{l}I \\ 0\end{array}\right)$. In particular, this is true for $X=V$. Since $\left(\begin{array}{l}I \\ 0\end{array}\right)$ is in $\operatorname{ker} D$, 
$U\left(\begin{array}{l}I \\ 0\end{array}\right)$ is in $\operatorname{ker} D$ as well, and therefore $U\left(\begin{array}{l}I \\ 0\end{array}\right)=\left(\begin{array}{l}r I \\ s I\end{array}\right)$ for some complex numbers $r$ and $s$. Thus

$$
U^{*} V U\left(\begin{array}{l}
I \\
0
\end{array}\right)=U^{*} V\left(\begin{array}{l}
r I \\
s I
\end{array}\right)=U^{*}\left(\begin{array}{l}
r V \\
s V
\end{array}\right) .
$$

The vector $\left(\begin{array}{l}r V \\ s V\end{array}\right)$ is in the span of the $2 \pi$ and $-2 \pi$ eigenspaces of $D$. The components of the eigenvectors of these two eigenspaces are linear combinations of operators of the form $V^{k} W^{l}$, where $k^{2}+l^{2}=1$. Therefore the components are linear combinations of $V^{ \pm 1}$ and $W^{ \pm 1}$. Furthermore, since $U^{*} V U\left(\begin{array}{l}I \\ 0\end{array}\right)=$ $\left(\begin{array}{l}Y \\ 0\end{array}\right)$ for some $Y$, we see that for some choice of complex numbers $a, b, c, d$,

$$
U^{*} V U\left(\begin{array}{l}
I \\
0
\end{array}\right)=\left(\begin{array}{c}
a V+b V^{-1}+c W+d W^{-1} \\
0
\end{array}\right)
$$

whence

$$
\phi(V)=a V+b V^{-1}+c W+d W^{-1} .
$$

Furthermore,

$$
\begin{aligned}
I= & \phi(V)(\phi(V))^{*} \\
= & \left(|a|^{2}+|b|^{2}+|c|^{2}+|d|^{2}\right) I \\
& +a \bar{b} V^{2}+\bar{a} b V^{-2}+\left(a \bar{c}+\lambda^{-1} \bar{b} d\right) V W^{-1}+(a \bar{d}+\lambda \bar{b} c) V W \\
& +\left(b \bar{d}+\lambda^{-1} \bar{a} c\right) V^{-1} W+(b \bar{c}+\lambda \bar{a} d) V^{-1} W^{-1}+c \bar{d} W^{2}+\bar{c} d W^{-2},
\end{aligned}
$$

which, upon inspection, shows that only one of $a, b, c, d$ is nonzero, and the one that is nonzero has absolute value 1 . Repeating the above argument with $W$ allows us to obtain a similar result for $\phi(W)$. If we additionally impose the condition $\phi(W) \phi(V)=\lambda \phi(V) \phi(W)$, then we are left with four families of possible elements of Iso $\left(A_{\theta}, \mathscr{H}, D\right)$. Specifically, for any two complex numbers $a$ and $b$ of modulus 1 , we have

$$
\begin{array}{ll}
\phi_{a, b}^{0}(V)=a V, & \phi_{a, b}^{1}(V)=a W^{-1}, \\
\phi_{a, b}^{0}(W)=b W, & \phi_{a, b}^{1}(W)=b V, \\
& \\
\phi_{a, b}^{2}(V)=a V^{-1}, & \phi_{a, b}^{3}(V)=a W, \\
\phi_{a, b}^{2}(W)=b W^{-1}, & \phi_{a, b}^{3}(W)=b V^{-1} .
\end{array}
$$

We now show that all of these automorphisms are in $\operatorname{Iso}\left(A_{\theta}, \mathscr{H}, D\right)$. Fix $a$ and $b$, and define $U: \mathscr{H} \longrightarrow \mathscr{H}$ by

$$
U\left(\begin{array}{c}
\mp \sqrt{k^{2}+l^{2}} V^{k} W^{l} \\
(l k+l) V^{k} W^{l}
\end{array}\right)=\left(\begin{array}{c}
\mp a^{-k} b^{-l} \sqrt{k^{2}+l^{2}} V^{k} W^{l} \\
a^{-k} b^{-l}(l k+l) V^{k} W^{l}
\end{array}\right) .
$$

Then $U$ commutes with $D$ and $U^{*} X U=\phi_{a, b}^{0}(X)$ for all $X$ in $A_{\theta}$. Next, for $j=1,2,3, \phi_{a, b}^{j}=\phi_{1,1}^{j} \phi_{a, b}^{0}$. Therefore, to show that all of the automorphisms above are in $\operatorname{Iso}\left(A_{\theta}, \mathscr{H}, D\right)$, we need only show that $\phi_{1,1}^{j}$ is in the isometry group. Furthermore, $\left(\phi_{1,1}^{1}\right)^{2}=\phi_{1,1}^{2}$ and $\left(\phi_{1,1}^{1}\right)^{3}=\phi_{1,1}^{3}$, so all that is left is to verify that $\phi_{1,1}^{1}$ is in $\operatorname{Iso}\left(A_{\theta}, \mathscr{H}, D\right)$. 
Define $U: \mathscr{H} \longrightarrow \mathscr{H}$ by

$$
U\left(\begin{array}{c}
\mp \sqrt{k^{2}+l^{2}} V^{k} W^{l} \\
(l k+l) V^{k} W^{l}
\end{array}\right)=\left(\begin{array}{c}
\mp l \lambda^{-l k} \sqrt{k^{2}+l^{2}} V^{-l} W^{k} \\
\lambda-l k(l k+l) V^{-l} W^{k}
\end{array}\right) .
$$

Straightforward computation yields that $U$ is unitary, commutes with $D$, and implements $\phi_{1,1}^{1}$.

Finally, we identify the group operation on these automorphisms. Define a homomorphism $\mu: \mathbb{Z}_{4} \longrightarrow \operatorname{Aut}\left(\mathbb{T}^{2}\right)$ by stipulating $\mu(1)(a, b)=\left(b^{-1}, a\right)$, and form the semidirect product $\mathbb{T}^{2} \rtimes_{\mu} \mathbb{Z}_{4}$, with the binary operation

$$
((a, b), j)((c, d), k)=(\mu(k)(a, b) \cdot(c, d), j+k) .
$$

The map $\phi_{a, b}^{k} \mapsto((a, b), k)$ from $\operatorname{Iso}\left(A_{\theta}, \mathscr{H}, D\right)$ to $\mathbb{T}^{2} \rtimes_{\mu} \mathbb{Z}_{4}$ is clearly a bijection. Moreover, $\phi_{a, b}^{0} \phi_{1,1}^{k}=\phi_{\mu(k)(a, b)}^{k}$ for $k=1,2,3$ and $a$ and $b$ in $\mathbb{T}$. Hence, $\phi_{a, b}^{j} \phi_{c, d}^{k}=\phi_{1,1}^{j} \phi_{a, b}^{0} \phi_{1,1}^{k} \phi_{c, d}^{0}=\phi_{1,1}^{j} \phi_{1,1}^{k} \phi_{\mu(k)(a, b)}^{0} \phi_{c, d}^{0}=$ $\phi_{1,1}^{j+k} \phi_{\mu(k)(a, b),(c, d)}^{0}=\phi_{\mu(k)(a, b),(c, d)}^{j+k}$, whence the map $\phi_{a, b}^{k} \mapsto((a, b), k)$ is a homomorphism. Therefore, we have proved

Theorem 3.1. For $0<\theta<1$, Iso $\left(A_{\theta}, \mathscr{H}, D\right) \cong \mathbb{T}^{2} \rtimes_{\mu} \mathbb{Z}_{4}$.

\section{THE $C^{*}$-ALgEBRA GENERATED BY THE UNILATERAL SHIFT}

The isometry groups that we have computed to this point have all been compact Lie groups. In this section, we give an example of an unbounded Fredholm module whose isometry group is neither compact nor a Lie group.

For each $k \in \mathbb{N}$, let $\delta_{k} \in l^{2}(\mathbb{N})$ denote the unit point mass at $k$, and let $S\left(\delta_{k}\right)=\delta_{k+1}$ be the unilateral shift operator on $l^{2}(\mathbb{N})$. Define $D: l^{2}(\mathbb{N}) \longrightarrow$ $l^{2}(\mathbb{N})$ by $D\left(\delta_{k}\right)=k \delta_{k}$. Then $\left(l^{2}(\mathbb{N}), D\right)$ is an unbounded Fredholm module over the $C^{*}$-algebra $C^{*}(S)$ generated by $S$.

Lemma 4.1. Let $\left\{a_{j}\right\}$ be a bounded sequence of complex numbers, and define an operator $X$ on $l^{2}(\mathbb{N})$ by $X\left(\delta_{k}\right)=a_{k} \delta_{k+1}$. Then $X$ is in $C^{*}(S)$ if and only if $\left\{a_{j}\right\}$ converges.

Proof. Suppose that $\left\{a_{j}\right\}$ converges to $a$. Then $X-a S$ is a compact operator and thus $X=a S+(X-a S)$ is in $C^{*}(S)$. Conversely, suppose $X$ is in $C^{*}(S)$. Then there exists a Toeplitz operator $T$ and a compact operator $K$ so that $X=T+K$. Now, for each $j \in \mathbb{N}, a_{j}=\left\langle X\left(\delta_{j}\right), \delta_{j+1}\right\rangle=\left\langle T\left(\delta_{j}\right), \delta_{j+1}\right\rangle+$ $\left\langle K\left(\delta_{j}\right), \delta_{j+1}\right\rangle$. Since $T$ is a Toeplitz operator, $\left\langle T\left(\delta_{j}\right), \delta_{j+1}\right\rangle=\left\langle T\left(\delta_{1}\right), \delta_{2}\right\rangle$ for all $j \in \mathbb{N}$. Since $K$ is compact, $\lim _{j \rightarrow \infty}\left\langle K\left(\delta_{j}\right), \delta_{j+1}\right\rangle=0$, so $\lim _{j \rightarrow \infty} a_{j}=$ $\left\langle T\left(\delta_{1}\right), \delta_{2}\right\rangle$ and $\left\{a_{j}\right\}$ converges.

Theorem 4.2. Let $\mathscr{X}$ denote the set

$$
\{f: \mathbb{N} \longrightarrow \mathbb{T}:\{f(n)\} \text { converges }\}
$$

and give $\mathscr{X}$ the topology induced by the metric

$$
d(f, g)=\sup _{n \in \mathbb{N}}|f(n)-g(n)| .
$$

Then $\operatorname{Iso}\left(C^{*}(S), l^{2}(\mathbb{N}), D\right)$, viewed as a topological space with the topology of pointwise convergence, is homeomorphic to $\mathscr{X}$.

Proof. Choose $\phi \in \operatorname{Iso}\left(C^{*}(S), l^{2}(\mathbb{N}), D\right)$, and let $U$ be a unitary on $l^{2}(\mathbb{N})$ that commutes with $D$ and implements $\phi$. For each $k \in \mathbb{N}$, the $k$-eigenspace of 
$D$ is spanned by $\delta_{k}$, and since $U$ commutes with the spectral projections of $D, U\left(\delta_{k}\right)=\lambda_{k} \delta_{k}$ for some complex number $\lambda_{k}$ of modulus 1 . Thus for each $k, \phi(S)\left(\delta_{k}\right)=U^{*} S U\left(\delta_{k}\right)=\lambda_{k} \bar{\lambda}_{k+1} \delta_{k}$, and by Lemma 4.1, the sequence $\left\{\lambda_{k} \bar{\lambda}_{k+1}\right\}$ converges. Define a map $\alpha: \operatorname{Iso}\left(C^{*}(S), l^{2}(\mathbb{N}), D\right) \longrightarrow \mathscr{Z}$ by $\alpha(\phi)(k)=\lambda_{k} \bar{\lambda}_{k+1}$. Since $\phi$ is completely determined by its value on $S$, $\alpha$ is an injection. Furthermore, given $f \in \mathscr{X}$, define a unitary $U$ on $\mathbb{N}$ by $U\left(\delta_{k}\right)=\left(\prod_{n=1}^{k-1} \overline{f(k)}\right) \delta_{k}$. Then the automorphism of $C^{*}(S)$ that is implemented by $U$ is mapped to $f$ by $\alpha$, and thus $\alpha$ is a bijection.

Finally, we check that $\alpha$ is a homeomorphism. A net $\left\{\phi_{\nu}\right\}$ in Iso $\left(C^{*}(S), l^{2}(\mathbb{N}), D\right)$ converges in the topology of pointwise convergence if and only if $\left\{\phi_{\nu}(S)\right\}$ converges in norm. Therefore, to show that $\alpha$ is a homeomorphism, it suffices to show that for any two elements $\phi$ and $\psi$ of Iso $\left(C^{*}(S), l^{2}(\mathbb{N}), D\right),\|\phi(S)-\psi(S)\|=d(\alpha(\phi), \alpha(\psi))$. Write $\phi(S)=$ $\sum_{k \in \mathbb{N}} a_{k} \delta_{k}, \psi(S)=\sum_{k \in \mathbb{N}} b_{k} \delta_{k}$. Then $\phi(S)-\psi(S)=\sum_{k \in \mathbb{N}}\left(a_{k}-b_{k}\right) \delta_{k}$, whence $\|\phi(S)-\psi(S)\|=\sup _{k \in \mathbb{N}}\left|a_{k}-b_{k}\right|=d(\alpha(\phi), \alpha(\psi))$.

\section{REFERENCES}

1. A. Connes, Compact metric spaces, Fredholm modules, and hyperfiniteness, Ergodic Theory Dynamical Systems 9 (1989), 207-220.

2. Alain Connes and John Lott, The metric aspect of noncommutative geometry, New Symmetry Principles in Quantum Field Theory (Cargèse, 1991), NATO Adv. Sci. Inst. Ser. B Phys., vol. 295, Plenum Press, New York, 1992, pp. 53-93.

3. S. Helgason, Differential geometry, Lie groups, and symmetric spaces, Academic Press, New York, 1978.

Department of Mathematics, Texas Christian University, Fort Worth, Texas 76129

E-mail address: epark@gamma. is.tcu.edu 